\title{
Platoon-based traffic flow model for estimating breakdown probability at single-lane expressway bottlenecks
}

\section{$\operatorname{AUTHOR}(\mathrm{S})$ :}

Shiomi, Yasuhiro; Yoshii, Toshio; Kitamura, Ryuichi

\section{CITATION:}

Shiomi, Yasuhiro ... [et al]. Platoon-based traffic flow model for estimating breakdown probability at single-lane expressway bottlenecks. Transportation Research Part B:

Methodological 2011, 45(9): 1314-1330

\section{ISSUE DATE:}

2011-11

URL:

http://hdl.handle.net/2433/152369

\section{RIGHT:}

(c) 2011 Elsevier Ltd.; この論文は出版社版でありません。引用の際には 出版社版をご確認ご利用ください。; This is not the published version. Please cite only the published version. 


\title{
Platoon-Based Traffic Flow Model for Estimating Breakdown Probability at Single-Lane Expressway Bottlenecks
}

\author{
Yasuhiro Shiomi $^{\mathrm{a}, 1 *}$, Toshio Yoshii ${ }^{\mathrm{b}}$ and Ryuichi Kitamura ${ }^{\mathrm{a}}$ \\ ${ }^{a}$ Department of Urban Management, Kyoto Univercity, Nishikyo-ku, Kyoto 615-8540, Japan \\ ${ }^{b}$ Department of Civil and Environmental Engineering, Ehime Univercity, Bunkyo-cho, Matsuyama 790-8577, Japan
}

\begin{abstract}
This study investigates the mechanism of traffic breakdown and establishes a traffic flow model that precisely simulates the stochastic and dynamic processes of traffic flow at a bottleneck. The proposed model contains two models of stochastic processes associated with traffic flow dynamics: a model of platoon formation behind a bottleneck and a model of speed transitions within a platoon. After these proposed models are validated, they are applied to a simple one-way, one-lane expressway section containing a bottleneck, and the stochastic nature of traffic breakdown is demonstrated through theoretical exercises.
\end{abstract}

Keywords: Traffic Breakdown; Breakdown Probability; Platoons; Desired Speed Distribution; Expressways Bottlenecks

\section{Introduction}

Traffic capacity plays an important role in various aspects of transportation analysis, including the planning, design, and operation of freeways. It is defined as the maximum number of vehicles that can pass a roadway section per unit of time under ideal conditions. This capacity concept is often confused with the breakdown flow rate, which is the flow rate at which traffic flow falls into breakdown and a congestion queue appears. Most traffic breakdowns appear before the volume reaches the traffic capacity. Several authors have demonstrated that the breakdown flow rate that induces a breakdown varies even under the same road and other environmental conditions (Elefteriadou et al., 1995; Minderhoud et al., 1997; Kühne and Anstett, 1999; Daganzo et al., 1999; Lorenz and Elefteriadou, 2000; Okamura et al., 2000). This may suggest another critical factor that precedes traffic breakdowns. Once a congestion queue appears, not only does the speed of the flow decrease, but the flow rate also decreases dramatically; therefore, it is extremely important to avoid traffic breakdown and maintain traffic flow in the free-flow state in terms of freeway operation and management. Thus, the precise nature of variability in traffic breakdown should be understood.

With respect to the mechanism of traffic breakdown, it has been noted that traffic flow breakdown at freeway bottlenecks is strongly related to platoons (e.g., Koshi, 1986; Mahnke et al., 2005). In particular, Koshi's conjecture is as follows: "a platoon is formed by slow vehicles that form moving bottlenecks. A vehicle with a higher desired speed catches up with a slow vehicle and is forced to follow it. The number of following vehicles behind the moving

\footnotetext{
* Corresponding author. Tel.: +81-75-383-3235; fax: +81-7-383-3236.
}

E-mail address: shiomi@trans.kuciv.kyoto-u.ac.jp 
bottleneck increases, generating a platoon in which traffic density is locally high. Once a vehicle within a platoon decelerates at a bottleneck section for some reason, the deceleration wave propagates upstream, and a traffic flow breakdown is likely to occur." In this sense, a large platoon can be regarded as a sort of metastable traffic flow condition (Kerner and Rehborn, 1997) that causes traffic breakdown. Several studies (Tamura and Chisyaki, 1987; Surasak et al., 2001) revealed that a platoon size distribution followed a monotonic decreasing function whose parameters were determined by traffic volume. Based on Koshi's conjecture, even when traffic volume is not very large, a large platoon can form with some probability before traffic breakdown. This process can be considered as one possible explanation of the variation in the breakdown flow rate.

Several studies have attempted to demonstrate the variable nature of traffic breakdown. Lorenz et al. (2000) and Okamura et al. (2000) defined the probability of traffic breakdown as the ratio of the number of traffic breakdown occurrences to the observation time. It is evaluated on the basis of classified levels of traffic flow rate, e.g., 5-min traffic counts. In addition, they investigated the relationship between flow rate and breakdown probability. Brilon et al. (2005), assuming that observation of traffic capacity is a censored observation, established a method of estimating the stochastic traffic capacity by applying survival analysis. Geistefeldt et al. (2009) compared two methodologies for empirically estimating the breakdown flow rate distribution: direct estimation of breakdown probabilities for groups of traffic volumes and estimation of breakdown flow rate distribution functions on the basis of statistical models of censored data. They concluded that the latter method yielded better results than the former. However, these studies adopted macroscopic or empirical approaches and assumed that the breakdown flow rate fluctuated randomly regardless of traffic flow conditions. The mechanism underlying the formation of traffic congestion was not well represented and understood in these studies.

Some studies analysed the variable nature of the breakdown flow rate microscopically and theoretically. For example, Laval (2006) analyzed the mechanism of fluctuations in breakdown flow rate by applying a modified kinematic wave theory in which slow vehicles were considered as moving bottlenecks that caused traffic breakdown. In that study, only two types of vehicle (having average and slow speeds) were considered for simplicity. Kühne et al. $(2005,2007)$ developed a master equation governing cluster evolution based on dynamical equations such as the balance equation and the Fokker-Planck equation. A cluster was defined as a congested state, and the master equation described the growth and decline of car clusters. A probability exceeding a threshold cluster size was defined as the breakdown probability. In the study, heterogeneity in driving behavior was not explicitly considered, but car clusters were assumed to form as a result of randomness in arrivals at a given bottleneck. In addition to random arrivals, heterogeneity in driving behaviors such as desired speeds and passing maneuvers may significantly influence the formation of car clusters and should be considered.

The present study investigates the mechanism of traffic breakdown and establishes a traffic flow model that precisely simulates the stochastic and dynamic processes of traffic flow at a bottleneck on the basis of the conjecture proposed by Koshi (1986). In particular, as a first stage for developing the model, this study targets only the traffic flow on single-lane sections of expressways. The proposed model contains two stochastic processes associated with traffic flow dynamics: a platoon formation model and a speed transition model within a platoon. In the former, platoon formation is assumed to be caused by variety in the desired speed of vehicles. This model represents platoon formation and estimates the distribution of platoon sizes in relation to traffic flow rate, roadway structural factors, and desired speed distribution. However, it is impossible to directly observe the desired speed distribution because vehicles catch up with the vehicle ahead and are forced to follow it. Thus, an inverse method of estimating the parametrically defined desired speed distribution from the observed platoons is also developed. On the other hand, the latter represents vehicle behavior in a platoon when it passes through a bottleneck. A following vehicle adjusts its acceleration and deceleration in response to the trajectory of the leading vehicle. Although the speed of the following vehicle is almost the same as that of the leading vehicle, this study assumes that the following vehicle tends to reduce its speed when platoons pass through a bottleneck section. On the basis of this assumption, speed transitions within a platoon at a bottleneck can be regarded as dynamic stochastic processes. Thus, the Markov process is assumed to be applicable to transitions in the speed of vehicles sequentially passing through the bottleneck. This model can estimate the probability of traffic breakdown when a platoon of a specific size passes through a bottleneck. Finally, the proposed models are combined to estimate the breakdown probability at a given traffic flow rate at a specific bottleneck section of a single-lane expressway.

This paper is organized as follows. In section 2, the criteria between following vehicles and others is clarified on the basis of the speed correlation between successive vehicles. In section 3, the platoon formation and speed transition models are developed, and the breakdown probability is numerically defined. In section 4 , the desired speed distribution, which is required in the platoon formation model as an input based on the observed traffic flow 
data, is estimated, and the proposed model is validated. In section 5, a model estimating breakdown probability with respect to traffic flow rate is applied to a simple one-way, one-lane expressway section containing a bottleneck, and the stochastic nature of traffic breakdown is then demonstrated through theoretical exercises.

\section{Definition of Platoons}

A platoon is generally defined as a group of vehicles traveling together, either voluntarily or involuntarily, because of signal control, geometrics, or other factors (HCM, 2000). In this study, a platoon on a single-lane section, where overtaking is impossible, is defined as a group consisting of a leading vehicle driving at its desired speed and following vehicles that cannot drive at their desired speed because of the vehicles ahead. In this section, the criteria for distinguishing between following vehicles and other vehicles are examined on the basis of fixed-point observation data.

\subsection{Methodology}

The difference between following vehicles and others is normally judged on the basis of time headway, distance headway, and/or relative speed. Time headway is the most widely accepted criterion; its threshold value is called the critical headway. Lay (1986) examined the notion of critical headway as defined by various authors and suggested three distinct states based on constraint conditions: when headway $<2.5$ [s], traffic is following; when headway is 2.5-9.0 [s], traffic is either following or free; and when headway > 9.0 [s], traffic is free. Bennet and Dunn (1994) suggested that both relative speeds and time headway are suitable platoon criteria. Thus, the present study determines the critical headway by focusing on relative speeds and traffic states.

Three types of driving state are defined: free moving, impeded, and transition. Leading vehicles and vehicles moving alone belong to the first state, and are defined as free-moving vehicles. Most following vehicles belong to the second state, and are defined as impeded vehicles. Furthermore, when a vehicle is catching up with a platoon ahead, the vehicle reduces its speed but is not following the vehicle ahead. These vehicles shifting from free moving to impeded belong to the third state, and are defined as transition vehicles. If the time headway between two successive vehicles is small, it can be definitely assumed that the vehicle behind is impeded by the vehicle ahead. In this case, the speeds of the two vehicles obtained by a fixed-point observation should be almost the same; that is, their relative speed is small, and their observed speeds show a significant correlation. When the time headway is slightly longer, free-moving vehicles appear in the vehicles behind, and the ratio of free-moving vehicles decreases as the time headway increases. At the same time, the variance in the relative speed should increase, and the correlation should decrease. Finally, if the time headway is sufficiently long, vehicles behind can drive freely unrestricted by those in front. As a result, the speeds of two successive vehicles should be independent, with the correlation between them close to 0 . Based on this consideration, the relationship between time headway and correlation coefficient in the speeds of two successive vehicles and the relationship between time headway and the component ratio of each driving state can be assumed, as illustrated in Figure 1. This figure shows that as time headway increases, the correlation decreases, and the ratio of transition vehicles to impeded vehicles increases. Then, at time headway $\mathrm{C}$, no vehicle is impeded. Therefore, in this study, the time headway corresponding to traffic state $\mathrm{C}$ in Figure 1 is defined as the critical time headway.

\subsection{Critical Time Headway between Free-Moving and Impeded Vehicles}

To validate the conjecture and determine the critical headway, the relationship between time headway and correlation coefficient is investigated using real field observation data. The observations were made at 72.6 kilometer post $(\mathrm{kp})$ of the Tokai-Hokuriku expressway from 13:00 to 19:00 on July 18, 2004, and from 13:00 to 17:00 on July 19, 2004, by using video cameras. The observation site is on a divided two-way, two-lane expressway section where it is physically impossible for faster vehicles to pass other vehicles (see Figure 2). The speed, time headway, and vehicle type of individual vehicles were obtained in the observations. Note that only traffic data for 


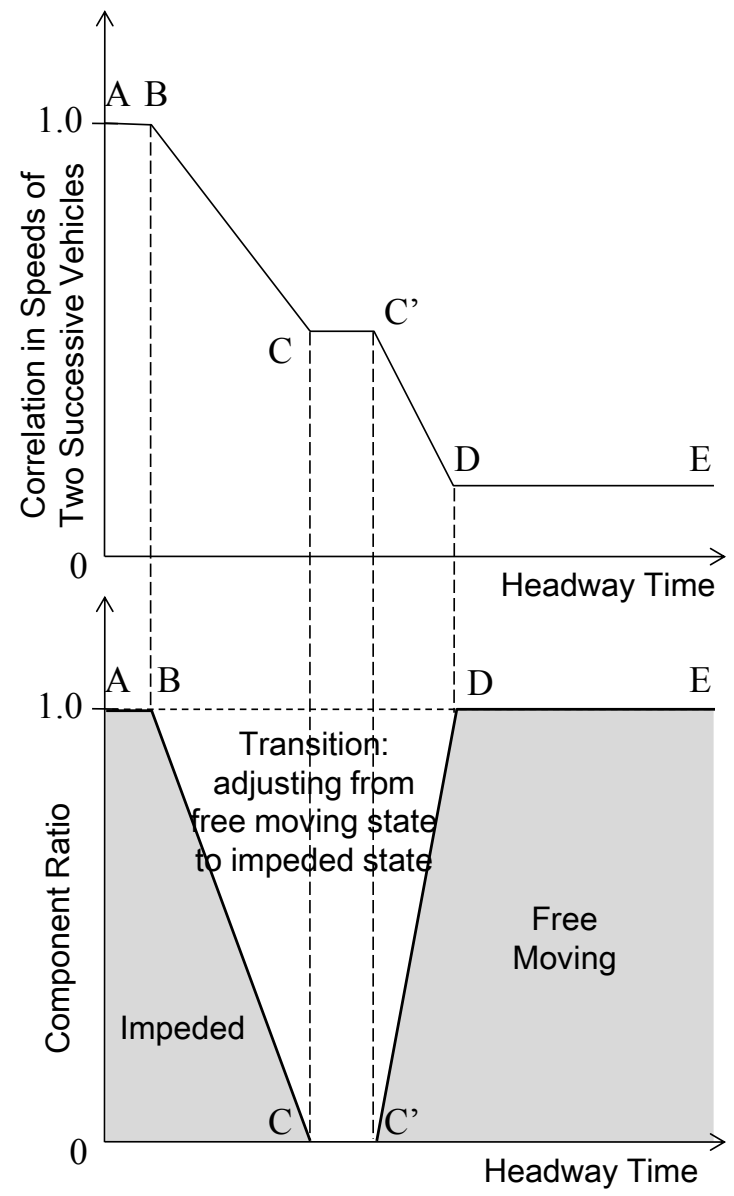

Figure 1. Assumed Relationships between Time Headway, Correlation in Speeds, and Component Ratio. Although the figure shows linear relationships, they do not have to be linear. The order in time headway of state C, where the component ratio of impeded vehicles becomes zero, and C', where the component ratio of free-moving vehicles becomes greater than zero, also does not have to be as shown.

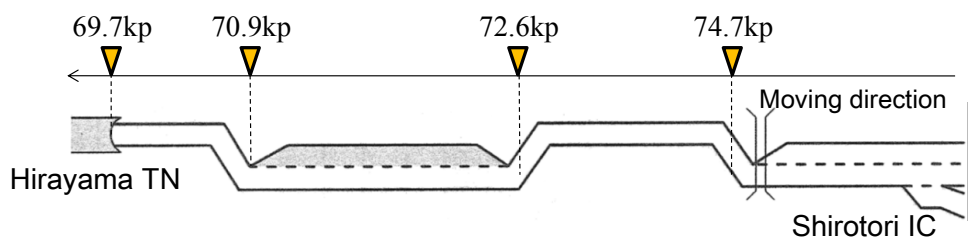

Figure 2. Observation Site on Tokai-Hokuriku Expressway. Additional lanes are occasionally available on the section upstream from $74.7 \mathrm{kp}$ and the section between $72.6 \mathrm{kp}$ and $70.9 \mathrm{kp}$ so that following vehicles can overtake leading vehicles. The entrance to the Hirayama tunnel at $69.7 \mathrm{kp}$ is a bottleneck. Observations were also made at $74.7 \mathrm{kp}$ and $69.7 \mathrm{kp}$ in the same period and by the same method as at $72.6 \mathrm{kp}$.

the free-flowing traffic state observed on non-rainy days in the daytime (between 9:00 and 16:00) were targeted.

The calculation results showing the relationship between time headway and correlation coefficient are summarized in Figure 3. This relationship is approximately piecewise-linear, as shown in Figure 1. Therefore, in this study, the time headway corresponding to traffic state $\mathrm{C}$ in Figure 3 is used as the critical headway. Vehicles driving with a headway shorter than the critical headway are defined as following vehicles, and those with a longer headway are defined as others. In particular, $4.0[\mathrm{~s}]$ is determined as the critical headway. 


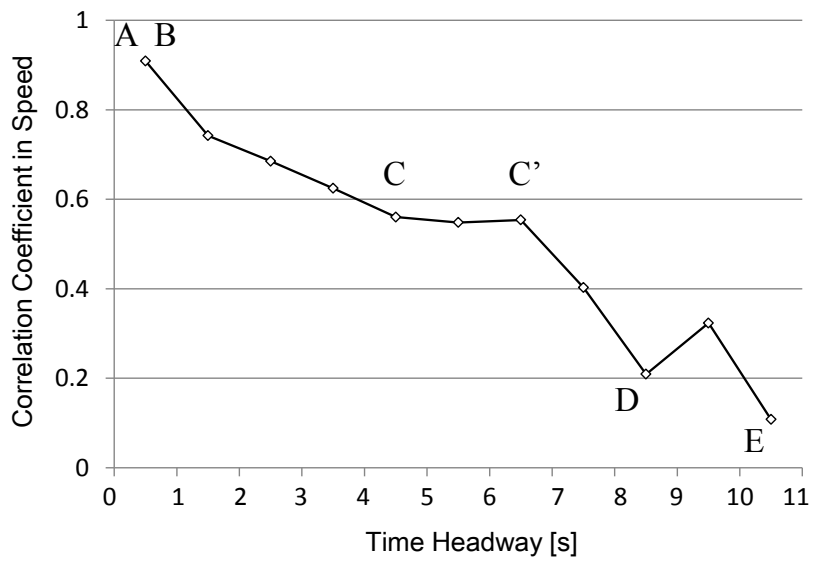

Figure 3. Relationship between Time Headway and Correlation Coefficient. The correlation coefficients are calculated for every $1[\mathrm{~s}]$ of time headway.

\section{Establishment of Platoon-Based Traffic Flow Model for Estimating Breakdown Probability}

In this section, a model for estimating the breakdown probability at an expressway bottleneck is developed. The focus is on bottlenecks on ordinary sections, such as sag sections or tunnel entrances, where traffic breakdown occurs because of fractional lowering of vehicle speed (Koshi et al., 1983). In this model, it is assumed that the complexity of traffic flow arises from the heterogeneity of the desired speeds of drivers and that platoons form stochastically because of the differences in desired speed as well as randomness in arriving at the target section. Moreover, when a large platoon passes through a bottleneck, a greater chance of breakdown is assumed. The traffic flow model based on this concept is established and defined as the Platoon-based traffic flow model. The model includes platoon formation and speed transition models. The breakdown probability with respect to the traffic flow rate is numerically formulated using the established models. Note that, for simplicity, the modeling was limited to a single-lane expressway.

\subsection{Definition of Breakdown Flow Rate and Breakdown Probability}

Traffic capacity is traditionally defined as the maximum number of vehicles that can pass a certain point in a certain time interval with given traffic conditions and roadway circumstances. To avoid terminological confusion and remain consistent with the traditional definition of traffic capacity, the breakdown flow rate is defined as the traffic flow rate inducing traffic breakdown. Moreover, the breakdown probability is defined as the probability of breakdown at a certain place when the traffic flow rate or platoon size is given. A certain traffic state is assumed to have some probability of breakdown. As mentioned above, the platoon size and traffic flow rate are considered to be the factors that should affect the breakdown probability. In particular, when the traffic flow rate exceeds the traditionally defined traffic capacity, the breakdown probability is expected to be 1.0.

\subsection{Platoon Formation Model}

To understand traffic phenomena, several bunching models have been proposed. Among them, the geometric distribution model (Miller, 1961), the Borel-Tanner distribution model (Tanner, 1961), and the Miller distribution model (Miller, 1961) have been widely accepted (e.g., Surasak et al., 2001). The model proposed by Tanner, however, did not represent vehicles' movement on expressways. The models of Miller were also based on the strict assumptions that vehicles joined platoons singly and independently. Gibbs (1977) proposed a bunching model in which a queue would merge with other queues and be dispersed from other queues randomly. The model, however, tended to overestimate the ratio of free-driving vehicles when the traffic volume is large. From another viewpoint, bunching can be understood as a queue behind a moving bottleneck. Several models simulating the behavior of a moving bottleneck have been proposed (e.g., Gazis and Herman, 1992; Newell, 1998; Daganzo and Laval, 2004). 
However, these models were based mainly on fundamental diagrams and depicted average traffic states. This study instead focuses on fluctuations and the probabilistic nature of traffic flow.

In this model, each vehicle is assumed to have an independent desired speed $v$, and the speed distribution, $f_{V}(v)$, is given. Vehicles travel at their own desired speeds unless they catch up with the vehicle ahead. After catching up, the vehicle immediately drops its speed to the speed of the vehicle ahead and follows the vehicle, maintaining a given headway. Figure 4 shows an example of vehicle trajectories under these assumptions. $L$ indicates the length of the studied single-lane section, and the end of the section is set at a point representing a bottleneck section. Let $t_{i}^{i n}$ be the given entrance time of the $i$ th vehicle ( vehicle $i$ ) and $\hat{t}_{i}^{\text {out }}$ be the imaginary exit time of vehicle $i$ if no breakdown appears at the bottleneck, which is written as

$$
\hat{t}_{i}^{\text {out }}=\left\{\begin{array}{cl}
t_{i}^{\text {out }} & (\text { if } i=0) \\
\max \left(t_{i}^{\text {out }}, \hat{t}_{i-1}^{\text {out }}+h_{i}\right) & (\text { if } i=1,2,3, \ldots)
\end{array}\right. \text {. }
$$

Here, $t_{i}^{\text {out }}$ indicates the exit time of vehicle $i$ when it moves continuously at its desired speed within the study section, which is calculated by

$$
t_{i}^{\text {out }}=t_{i}^{\text {in }}+L / v_{i}
$$

and $h_{i}$ indicates the given time headway of vehicle $i$ when it follows the vehicle ahead. Moreover, $i=0$ indicates the boundary condition where no vehicles appear in front of it. In Figure 4, vehicle $i$ 's desired speed is so low that three vehicles follow it, and a platoon of four vehicles is formed. Note that a free-moving vehicle that moves alone, e.g., vehicle $i-1$, is also counted as a platoon of one vehicle.

Let $p\left(A_{v}\right)$ be the probability that a vehicle with desired speed $v$ becomes the lead vehicle of a platoon, $A_{v}$ be this event, and $p\left(k \mid A_{v}\right)$ be the conditional probability that a platoon of $k$ vehicles is formed given the occurrence of event $A_{v}$. Then, the probability $p^{p l t}(k)$ that a certain vehicle becomes the lead vehicle of a platoon of $k$ vehicles at the exit of the study section is written as

$$
p^{p l t}(k)=\int_{0}^{\infty} p\left(A_{v}\right) \cdot p\left(k \mid A_{v}\right) \cdot f_{V}(v) d v .
$$

Both $p\left(A_{v}\right)$ and $p\left(k \mid A_{v}\right)$ are calculated using the desired speed distribution. To remain in the free-moving state, vehicle $i$ must not catch up with any platoon that ends in vehicle $i-1$. Suppose that vehicle $i-1$ is the rear of a platoon whose lead vehicle is vehicle $i-k$; the necessary and sufficient condition for vehicle $i$ to remain in the freemoving state within the study section is

$$
t_{i}^{\text {out }}>t_{i-k}^{\text {out }}+\sum_{j=i-k+1}^{i} h_{j}, \forall k=1,2,3, \cdots,
$$

which can be rearranged into

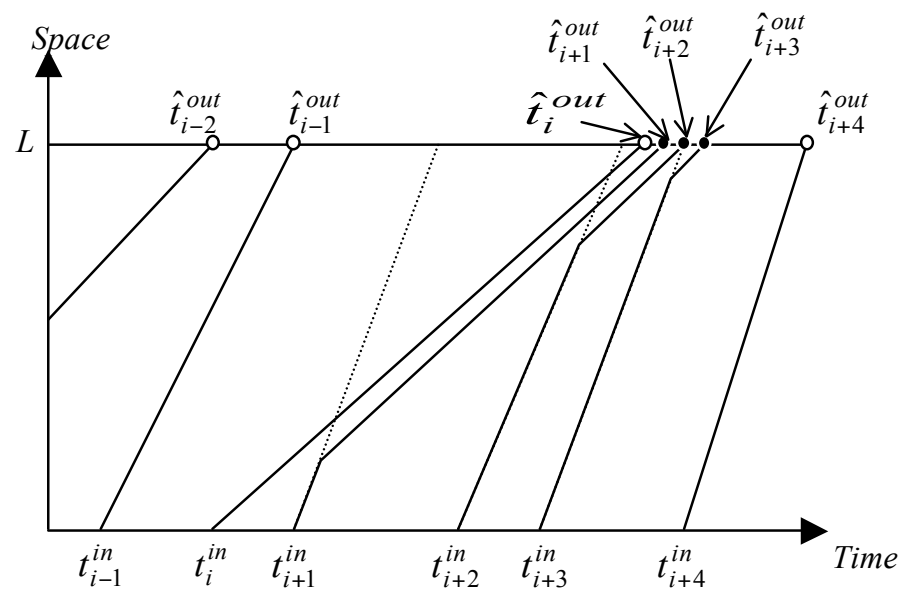

Figure 4. Trajectories of Traffic Flow. Solid lines indicate real vehicle trajectories, dotted lines indicate the trajectories of vehicles driving at their desired speed, open circles indicate freely driving vehicles in the study section, and solid circles indicate following vehicles. 


$$
v_{i-k}>\frac{L}{L / v_{i}+\left\{\left(t_{i}^{i n}-t_{i-k}^{i n}\right)-\sum_{j=i-k+1}^{i} h_{j}\right\}} \equiv d_{i, i-k}\left(v_{i}\right) .
$$

Then, the probability that a vehicle with desired speed $v$ can remain in the free-moving state is

$$
p\left(A_{v_{i}}\right)=\prod_{k=1}^{\infty} \operatorname{Pr}\left[v_{i-k}>d_{i, i-k}\left(v_{i}\right)\right] .
$$

By rearranging Eq. (6), $p\left(A_{v i}\right)$ can be expressed in relation to the desired speed distribution as

$$
p\left(A_{v_{i}}\right)=\prod_{k=1}^{\infty}\left[\int_{d_{i, i-k}\left(v_{i}\right)}^{\infty} f_{V}(u) d u\right] .
$$

Now, $p\left(k \mid A_{v}\right)$ will be derived. A platoon of $k$ vehicles is formed when $k-1$ vehicles form a queue just behind the lead vehicle, and the $k$ th vehicle behind the lead vehicle does not follow the $(k-1)$ th vehicle. Suppose that vehicle $i$ is the lead vehicle of a platoon. The necessary condition for vehicle $i+j$ to catch up with the platoon is

$$
t_{i+j}^{\text {out }} \leq t_{i}^{\text {out }}+\sum_{l=i+1}^{i+j} h_{l}
$$

which can be rearranged into

$$
v_{i+j} \geq \frac{L}{L / v_{i}-\left\{\left(t_{i+j}^{i n}-t_{i}^{i n}\right)-\sum_{l=i+1}^{i+j} h_{l}\right\}} \equiv e_{i, i+j}\left(v_{i}\right) .
$$

Then, the probability of a platoon of $k$ vehicles forming behind vehicle $i$ given the occurrence of event $A_{v_{i}}$ can be shown as

$$
p\left(k \mid A_{v_{i}}\right)=\left[\prod_{j=1}^{k-1} \operatorname{Pr}\left[t_{i+j}^{\text {out }} \leq t_{i}^{\text {out }}+\sum_{l=i+1}^{i+j} h_{l}\right]\right] \cdot \operatorname{Pr}\left[t_{i+k}^{\text {out }}>t_{i}^{\text {out }}+\sum_{l=i+1}^{i+k} h_{l}\right] .
$$

The first term on the right-side indicates the probability of $k-1$ vehicles forming a platoon, and the second term indicates the probability of the $k$ th vehicle from the lead vehicle being unable to catch up with the platoon. By substituting Eq. (9) into Eq. (10), $p\left(k \mid A_{v}\right)$ can be expressed as

$$
p\left(k \mid A_{v_{i}}\right)=\left[\prod_{j=1}^{k-1}\left\{\int_{e_{i, i+j}\left(v_{i}\right)}^{\infty} f_{V}(u) d u\right\}\right] \cdot\left[\int_{0}^{e_{i, i+k}\left(v_{i}\right)} f_{V}(u) d u\right] .
$$

Thus, by integrating Eqs. (7) and (11) with Eq. (3), $p^{p l t}(k)$, the probability that a certain vehicle becomes the lead vehicle of a platoon of $k$ vehicles at the exit of the study section, can be expressed in relation to the desired speed distribution. Then, the probability of a platoon of $k$ vehicles can be estimated as the ratio of the probability of a lead vehicle of a platoon of $k$ vehicles to the probability of a lead vehicle of a platoon of any size. That is, given each vehicle's entrance time and desired speed distribution in Eq. (12), the platoon size distribution $f_{S}(k)$ can be estimated as follows:

$$
f_{S}(k)=\frac{\int_{0}^{\infty} p\left(A_{u}\right) \cdot p\left(k \mid A_{u}\right) \cdot f_{V}(u) d u}{\int_{0}^{\infty} p\left(A_{u}\right) \cdot f_{V}(u) d u} .
$$

Simultaneously, the probability density $f_{P}\left(A_{v}, k\right)$ that a platoon whose lead vehicle's speed is $v$ and size is $k$ is generated can be written as

$$
f_{P}\left(A_{v}, k\right)=f_{V}(v) \cdot p\left(A_{v}\right) \cdot p\left(k \mid A_{v}\right) .
$$




\subsection{Speed Transition Markov Chain Model}

\subsubsection{Relationship between the Speeds of Successive Vehicles in a Platoon}

With respect to the mechanism of traffic breakdown at an expressway bottleneck, Koshi (1986) noted that when a large platoon goes through a bottleneck section, some vehicles within the platoon slow down slightly, which generates a deceleration wave, and eventually traffic breakdown occurs. On the other hand, the characteristics of platoons that cause traffic breakdown are not well understood. Oguchi et al. (2001) showed that a large platoon tends to, but does not always, cause traffic breakdown. It can be conjectured that when a platoon passes through a bottleneck, speed reduction occurs stochastically. If the platoon is small, even if speed reduction occurs, a deceleration wave will rarely propagate upstream. On the other hand, when the platoon is large, the deceleration wave propagates upstream, and traffic breakdown occurs.

In this study, this wave propagation is considered as a transition in the speed of a vehicle in the platoon at a specific observation point. As mentioned in 2.2, following vehicles, i.e., vehicles composing a platoon, are defined as those whose speeds are strongly correlated with the speed of the lead vehicle. Hence, the speed of a following vehicle is essentially almost identical to the speed of the leading vehicle. Because of instability in the following behavior, however, some fluctuation can be observed. Here, let $v_{i}$ and $v_{i+1}$ indicate the observed speeds of two successive vehicles $i$ and $i+1$ within a platoon. The relationship between $v_{i}$ and $v_{i+1}$ can be expressed as $v_{i+1}=v_{i}+$ $r_{i+1}$, where $r_{i+1}$ denotes the relative speed and indicates the fluctuation in the speed transition from vehicle $i$ to vehicle $i+1$. If the relative speed tends to be negative, it can be interpreted as a tendency toward deceleration in the platoon, which causes breakdown stochastically. Thus, to reveal when the relative speed becomes negative, the data observed at point $69.7 \mathrm{kp}$ in Figure 2 are used to investigate the relationship between the lead vehicle speed $v_{i}$ and the relative speeds of following vehicles $r_{i+1}$ within a platoon. As shown in Figure 5, as the speed of front vehicles is higher, the relative speeds tend to be lower. When the speed is around $80[\mathrm{~km} / \mathrm{h}]$, the relative speeds are distributed in a relatively positive area. On the other hand, when the speeds are less than $60[\mathrm{~km} / \mathrm{h}]$ in a single-lane section, the relative speeds are distributed around zero. This tendency must be related to each vehicle's desired speed; that is, if the speed of a front vehicle is high, the next vehicle's desired speed is likely to be lower, and the relative speed tends to be negative. In contrast, if the speed of a front vehicle is not very high, the next vehicle's desired speed is likely to be higher, and the relative speed tends to be positive. Furthermore, if the front vehicles' speeds are sufficiently low, the next vehicle must follow it; as a result, the relative speeds centralize around zero. This tendency also appears in the data in Table 1, which summarizes the means and standard deviations of the relative speeds as calculated in 10 $[\mathrm{km} / \mathrm{h}]$ increments of the front vehicle's speed. As a result, it is shown that the mean of relative speed varies according to the front vehicle's speed. In other words, the tendency of speed transition varies in accordance with the front vehicle's speed, and when the front vehicle's speed is less than $60[\mathrm{~km} / \mathrm{h}]$, a tendency toward deceleration in a platoon can be observed.

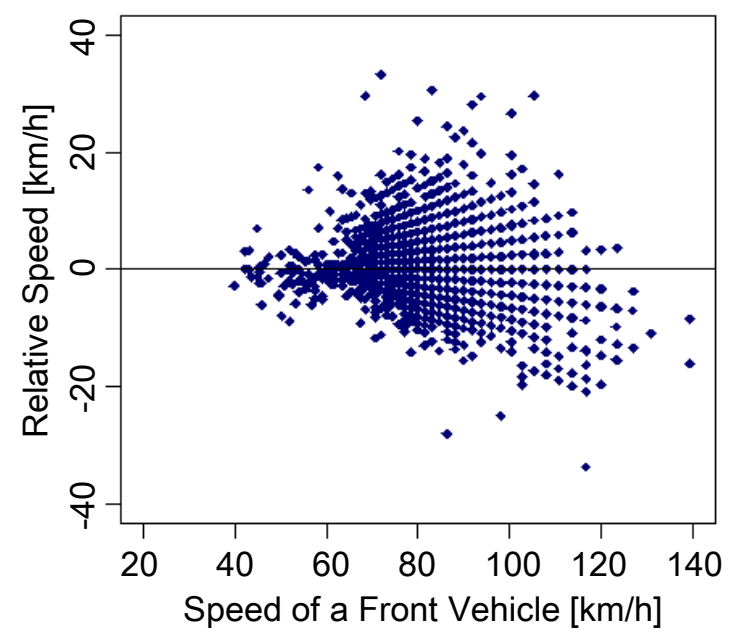

Figure 5. Relationship between Speed of the Leading Vehicle and Relative Speed in a Platoon. 
Table 1. Mean and Standard Deviation of Relative Speed.

\begin{tabular}{c|r|r|r|r|r|r|r|r}
\hline $\begin{array}{c}\text { Levels of Front } \\
\text { Vehicle Speed }\end{array}$ & \multicolumn{1}{|c|}{$40-50$} & \multicolumn{1}{|c|}{$50-60$} & $60-70$ & $70-80$ & $80-90$ & $90-100$ & $100-110$ & $110-$ \\
\hline $\mathrm{N}$ & 19 & 49 & 162 & 759 & 1,539 & 1,001 & 296 & 19 \\
Mean $[\mathrm{km} / \mathrm{h}]$ & -0.35 & -0.32 & 1.49 & 1.05 & 0.23 & -0.66 & -1.83 & -6.04 \\
S.D. $[\mathrm{km} / \mathrm{h}]$ & 3.18 & 4.48 & 4.77 & 4.74 & 4.94 & 5.15 & 6.68 & 6.00 \\
\hline
\end{tabular}

\subsubsection{Testing the Markov Property in Speed Transitions}

On the basis of the conjecture about the speed reduction mechanism discussed in 3.3.1, a hypothesis regarding speed transitions in a platoon is made:

Hypothesis: The transition in the speeds of vehicles propagating in a platoon passing a bottleneck sequentially has the Markov property.

To test the hypothesis, let $S=\left\{S_{0}, S_{1}, S_{2}, \ldots, S_{n}\right\}$ indicate the speed levels, $\left\{X_{t}, t=1,2, \ldots, n_{p}\right\}$ indicate the stochastic process of the speed levels of the $t$ th vehicle in a platoon consisting of $n_{p}$ vehicles, $m_{i}$ show the number of observations in which $t$ th vehicle $\left(t=1,2,3, \ldots, n_{p}-1\right)$ moves at the speed level $S_{i}, m_{i j}$ show the number of observations in which the speed levels of successive $t$ th and $t+1$ th vehicles change in the order of $S_{i}$ and $S_{j}$, and similarly $m_{i j}(\Delta)$ show the number of observations in which the speed levels of $t-\Delta$ and $t$ th vehicles $(t=\Delta+1$, $\left.\Delta+2, \ldots, n_{p}\right)$ change in the order of $S_{i}$ and $S_{j}$, and $m_{i j k}(\Delta)$ show the number of observations in which the speed levels of $t-\Delta, t$ and $t+1$ th vehicles $\left(t=\Delta+1, \Delta+2, \ldots, n_{p}-1\right)$ change in the order of $S_{i}, S_{j}$, and $S_{k}$. Here, $P_{i j}$ are defined as

$$
P_{i j}=\operatorname{Pr}\left[X_{t+1}=S_{j} \mid X_{t}=S_{i}\right\rfloor, \quad t=2,3, \ldots, n_{p}-1,
$$

which are estimated by the observations as $\hat{P}_{i j}=m_{i j} / m_{i}$. In the same way, $P_{i j k}(\Delta)$ are defined as

$$
P_{i j k}(\Delta)=\operatorname{Pr}\left[X_{t+1}=S_{k} \mid X_{t-\Delta}=S_{i}, X_{t}=S_{j}\right\rfloor, \quad t=\Delta+1, \Delta+2, \ldots, n_{p}-1,
$$

which are estimated by the observations as $\hat{P}_{i j k}(\Delta)=m_{i j k}(\Delta) / m_{i j}(\Delta)$.

If the hypothesis is valid, $P_{j k}=P_{i j k}(\Delta)$ should be valid for $\forall \Delta$. Thus, we set the null hypothesis

$$
\mathrm{H}_{0}: P_{j k}=P_{i j k}(\Delta) \text { for } \forall \Delta \text {. }
$$

The statistical value $\lambda(\Delta)$ is defined as

$$
\lambda(\Delta)=\prod_{i, j, k}\left\{\frac{\hat{P}_{j k}}{\hat{P}_{i j k}(\Delta)}\right\}^{m_{i j k}(\Delta)} .
$$

Then, under the null hypothesis $\mathrm{H}_{0}$, Eq. (17) follows the chi-squared distribution with $n(n+1)$ degrees of freedom (Anderson and Goodman, 1957).

$$
-2 \log \lambda(\Delta)=2 \sum_{i=0}^{n} \sum_{j=0}^{n} \sum_{k=0}^{n} m_{i j k}(\Delta) \cdot\left(\log \hat{P}_{i j k}(\Delta)-\log \hat{P}_{j k}\right) .
$$

Hereafter, the hypothesis will be tested using the data observed at $69.7 \mathrm{kp}$ on the Tokai-Hokuriku expressway, which is located just behind a bottleneck, as shown in Figure 2. The driving speed of each vehicle is classified into one of eight levels; less than $40[\mathrm{~km} / \mathrm{h}], 40-50[\mathrm{~km} / \mathrm{h}], 50-60[\mathrm{~km} / \mathrm{h}], 60-70[\mathrm{~km} / \mathrm{h}], 70-80[\mathrm{~km} / \mathrm{h}], 80-90[\mathrm{~km} / \mathrm{h}]$, 90-100 [km/h], and more than $100[\mathrm{~km} / \mathrm{h}]$. By using the speed data for following vehicles in platoons, the speed transition probabilities $\hat{P}_{j k}$ and $\hat{P}_{i j k}(\Delta)$ are estimated with respect to $\Delta=1$ and 2 . Note that the free-flow situation, in which the 5 -min speed average is greater than $40[\mathrm{~km} / \mathrm{h}]$, is targeted, and $\Delta$ is set to 1 or 2 , which means that car following behavior is assumed to be affected by at most two or three vehicles ahead. As a result, $-\log \lambda(1)=49.6$ and $-\log \lambda(2)=46.8$, whereas the $5 \%$ value of $\chi^{2}$ with 56 degrees of freedom is 74.5 . For both $\Delta=1$ and $\Delta=2$, the null hypothesis cannot be rejected at the $5 \%$ significance level. Thus, it can be concluded that the Markov property of speed transition in a platoon cannot be rejected; i.e., the hypothesis can be justified. 


\subsubsection{Model Formulation}

Assuming the Markov property of speed transitions in a platoon, the speed of vehicle $i+1, v_{i+1}$, is stochastically determined with respect to the front vehicle's speed $v_{i}$. Thus, the relative speed $r_{i+1}$ can be interpreted as stochastically determined solely by $v_{i}$. The stochastic model of the speed transition in this study has a state space comprising $\left\{S_{0}, S_{1}, \ldots, S_{n}\right\} . S_{0}$ is the state in which the speed of the vehicle passing the bottleneck is within the interval $\left[0, V_{0}\right] . S_{i}(i=1,2, \ldots, n-1)$ is that in which the speed is within the interval $\left(V_{i-1}, V_{i}\right] . S_{n}$ is that in which the speed is within the interval $\left(V_{n-1},+\infty\right]$, where $V_{0}, V_{1}, \ldots, V_{n-1}$ are predetermined real numbers. The state-to-state transition matrix $\mathbf{P}$ associated with this state space is defined as

$$
\mathbf{P}=\left(\begin{array}{cccc}
p_{S_{0} S_{0}} & p_{S_{0} S_{1}} & \cdots & p_{S_{0} S_{n}} \\
p_{S_{1} S_{0}} & p_{S_{1} S_{1}} & \cdots & p_{S_{1} S_{n}} \\
\vdots & \vdots & \ddots & \vdots \\
p_{S_{n} S_{0}} & p_{S_{n} S_{1}} & \cdots & p_{S_{n} S_{n}}
\end{array}\right)
$$

where $p_{A B}$ is the transition probability from state $A$ to $B$. Given the speed of the lead vehicle of a platoon $v_{t o p}$, the state vector $\mathbf{q}^{(i)}\left(v_{\text {top }}\right)$, which represents the state vector of the $i$ th vehicle from the lead vehicle in a platoon, is written as

$$
\mathbf{q}^{(i)}\left(v_{\text {top }}\right)=\mathbf{P}^{i} \cdot \mathbf{q}^{(0)}\left(v_{\text {top }}\right) \text {, }
$$

where

$$
\begin{gathered}
\mathbf{q}^{(0)}\left(v_{\text {top }}\right)=\left(\delta_{0}, \delta_{1}, \ldots, \delta_{n}\right)^{T}, \\
\delta_{i}=\left\{\begin{array}{l}
1\left(\text { if } v_{\text {top }} \in S_{i}\right) \\
0 \text { (otherwise }) .
\end{array}\right.
\end{gathered}
$$

\subsection{Breakdown Probability with Respect to Traffic Flow Rate}

Traffic breakdown is a transition from a free-flow traffic state to a congested traffic state. After breakdown, the traffic speed drops dramatically, and the discharge flow rate also decreases. Thus, in this study, a considerable traffic slowdown is assumed to indicate that traffic breakdown has occurred. Concretely, traffic breakdown is defined as follows.

Definition: Traffic breakdown occurs when a vehicle's speed in a platoon reaches the speed level $S_{0}$.

Moreover, it is assumed that once traffic breakdown occurs in a platoon passing through a bottleneck, congested flow is maintained at least until the entire platoon has passed through the bottleneck. Hence, the speed transition matrix in Eq. (18) can be rewritten as the absorbing Markov chain model shown in Eq. (20).

$$
\mathbf{P}=\left(\begin{array}{cccc}
1 & 0 & \cdots & 0 \\
p_{S_{1} S_{0}} & p_{S_{1} S_{1}} & \cdots & p_{S_{1} S_{n}} \\
\vdots & \vdots & \ddots & \vdots \\
p_{S_{n} S_{0}} & p_{S_{n} S_{1}} & \cdots & p_{S_{n} S_{n}}
\end{array}\right) .
$$

Hence, the platoon breakdown probability, that is, the probability that traffic breakdown occurs as a platoon passes through a bottleneck, can be calculated. Suppose that a platoon is composed of $k$ vehicles, and its lead vehicle's speed is $v_{t o p}$. Then, the platoon breakdown probability $p_{b d}^{p l t}\left(v_{\text {top }}, k\right)$ is written as

$$
p_{b d}^{p l t}\left(v_{t o p}, k\right)=\left\langle\mathbf{e}_{1}^{T}, \mathbf{P}^{k-1} \cdot \mathbf{q}^{(0)}\left(v_{t o p}\right)\right\rangle,
$$

where the operator $<\mathbf{A}, \mathbf{B}>$ indicates the dot product of vectors $\mathbf{A}$ and $\mathbf{B}$, and $\mathbf{e}_{1}$ indicates a unit vector $(1,0, \ldots, 0)$.

By combining the platoon breakdown probability with the platoon formation model which describes the probability that a platoon of size $k$ with a lead vehicle speed $v_{t o p}$ is generated, the breakdown probability $p_{b d}(Q)$, 
which is defined as the expectation of platoon breakdown probability given traffic flow rate $Q$ [veh/h], and the parameter vectors of the desired speed distribution $\boldsymbol{\beta}$ and the speed transition matrix $\mathbf{P}$ can be formulated as follows.

$$
p_{b d}(Q \mid \boldsymbol{\beta}, \mathbf{P})=\int \sum_{k} f_{P}\left(A_{v}, k \mid Q, \boldsymbol{\beta}\right) \cdot p_{b d}^{p l t}(v, k \mid \mathbf{P}) d v .
$$

\section{Estimation of Desired Speed Distribution}

In the platoon formation model proposed in 3.2, the desired speed distribution is required as an input parameter. The desired speed distribution is usually estimated as (i) the speed distribution of only the leading vehicles and/or isolated vehicles or (ii) the speed distribution of all vehicles under the condition that traffic volume is extremely low and every vehicle can drive at its own desired speed (TRB, 2000). However, direct observations inevitably contain biases. The speed distribution described in (i) tends to underestimate the desired speed distribution; regarding that described in (ii), the traffic flow under extremely low traffic volume is totally different from that under nearly congested conditions.

Several studies have proposed methods of estimating the desired speed distribution (e.g., Branston, 1979; Botma et al., 2001; Hoogendoorn, 2005), which are based on observations at a single site. In contrast, this study establishes a method of estimating the desired speed distribution using observations at two sites.

\subsection{Methodology}

The proposed method requires two-site observations. Vehicle arrival time data are obtained at a site located upstream along the target roadway section, and data on platoons are obtained at a site located downstream. Both data sets are used to estimate the desired speed distribution.

Given the desired speed distribution specified by a parameter vector $\beta$ and the observed vehicle arrival times $\widetilde{\mathbf{t}}^{i n}$ at the upstream point, the probability density $f_{P}\left(A_{v}, k \mid \boldsymbol{\beta}, \widetilde{\mathbf{t}}^{\text {in }}\right)$ of appearance of a platoon of size $k$ with lead vehicle speed $v$ can be calculated using Eq. (13). Then, the likelihood of the observation of platoons at the downstream point can be expressed as

$$
L(\boldsymbol{\beta})=\prod_{i=1}^{N} f_{P}\left(A_{\widetilde{v}_{i}}, \widetilde{k}_{i} \mid \boldsymbol{\beta}, \widetilde{\mathbf{t}}^{\text {in }}\right),
$$

where $N$ is the number of observed platoons, $\widetilde{v}_{i}$ is the speed of the lead vehicle of the $i$ th observed platoon, and $\tilde{k}_{i}$ is the size of the $i$ th observed platoon. By maximizing the likelihood function shown in Eq. (23), $\hat{\boldsymbol{\beta}}$ can be estimated as

$$
\hat{\boldsymbol{\beta}}=\arg \max L(\boldsymbol{\beta})=\arg \max \widetilde{L}(\boldsymbol{\beta}),
$$

where

$$
\widetilde{L}(\boldsymbol{\beta})=\ln L(\boldsymbol{\beta})=\sum_{i=1}^{N} \ln f_{P}\left(A_{\widetilde{v}_{i}}, \widetilde{k}_{i} \mid \boldsymbol{\beta}, \widetilde{\mathbf{t}}^{i n}\right)
$$

\subsection{Data Description}

The data were collected by video cameras at two different points on a divided two-lane, two-way section of the inbound Tokai-Hokuriku expressway, shown in Figure 2: one is at $74.7 \mathrm{kp}$, the entrance point of a single-lane section that is $2.1-\mathrm{km}$ long, and the other is at $72.6 \mathrm{kp}$, its exit point. Overtaking was prohibited in the study section. Two data sets were used for the analysis: one was observed from 14:30 to 15:30 on July 18, 2004, when the traffic volume was 657 [veh], and the other was observed from 14:45 to 15:45 on July 19, 2004, when the traffic volume was 1,123 [veh], just after the observation traffic became congested. Because both observations were made during the daytime on a holiday with no rain, it is conceivable that the attributes of vehicles composing the traffic flow were almost the same. The time when vehicles enter the study section was obtained at $74.7 \mathrm{kp}$, whereas the time 
headway and driving speed of each vehicle were obtained at $72.6 \mathrm{kp}$. In this study, it is assumed that differences in vehicle type do not affect platoon formation.

\subsection{Condition Settings for Estimating Desired Speed Distribution}

In this study, the desired speed distribution is assumed to fit a Gumbel distribution because this distribution can reduce the computational tasks required to calculate its probability distribution function. Hereafter, $\eta$ and $\mu$ denote the location and scale parameters of the Gumbel distribution, respectively. The following time headways in platoons are assumed to be the same among all vehicles. The average time headway of following vehicles observed at $72.6 \mathrm{kp}$, $h_{\text {ave }}$, is used as the following headway of all vehicles in this model. When the traffic volume was 657 [veh], $h_{\text {ave }}$ was calculated as 2.13 [s], and when the traffic volume was 1,123 [veh], $h_{\text {ave }}$ was calculated as 1.96 [s]. For estimating the desired speed distribution by using the observed arrival time of each vehicle at $74.7 \mathrm{kp}, f_{P}\left(A_{\tilde{v}}, \widetilde{k} \mid \boldsymbol{\beta}, \widetilde{\mathbf{t}}^{\text {in }}\right)$ in Eq. (25) cannot be analyzed numerically; therefore, the following approximation method is adopted.

Step 1: Desired speeds are randomly assigned to all the observed vehicles based on a desired speed distribution.

Step 2: In accordance with Eq. (1), the arrival time at the exit of the study section is calculated for each vehicle.

Step 3: All platoons derived as a result of Step 2 are classified according to the speed level of their lead vehicle, $S_{i}$ $(i=0,1, \ldots, n)$, and size, $k$. Let $R\left(S_{i}, k\right)$ denote the occurrence frequency of each type of platoon.

Step 4: By repeating Steps 1 to 3 10,000 times with a different random number sequence, the probability density $f_{P}\left(A_{v}, k\right)$ is approximately estimated using Eq. (26).

$$
f_{P}\left(A_{v}, k\right) \approx \frac{R\left(S_{i}, k\right)}{10,000 \cdot q}, i=0,1, \ldots, n,
$$

where $v \in S_{i}$ and $q$ indicates the observed traffic volume [veh].

To find $\hat{\boldsymbol{\beta}}$, all $\widetilde{L}(\boldsymbol{\beta})$ in the $\eta-\mu$ field where $80.0 \leq \eta \leq 110.0$ and $0.05 \leq \mu \leq 0.15$ were investigated at intervals of $\Delta \eta=0.1$ and $\Delta \mu=0.001$ in both cases.

\subsection{Estimation of Desired Speed Distribution}

To compare the proposed method to the methods in the literature, the desired speed distribution were estimated using the method of Botma et al. (2001), as well as the proposed method above. In the former method, the desired speed distribution $f(v \mid \boldsymbol{\theta})$ was estimated by deriving the $\hat{\boldsymbol{\theta}}$ value that maximized the likelihood function $L(\boldsymbol{\theta})$ as

$$
L(\boldsymbol{\theta})=\prod_{i=1}^{N}\left[f\left(v_{i} \mid \boldsymbol{\theta}\right)\right]^{\delta_{i}} \cdot\left[\int_{v_{i}}^{\infty} f(u \mid \boldsymbol{\theta}) d u\right]^{1-\delta_{i}},
$$

where $N$ denotes the number of observed vehicles, $v_{i}$ denotes the observed speed of the $i$ th vehicle, and $\delta_{i}$ denotes the driving state of the $i$ th vehicle; $\delta_{i}=0$ for a following vehicle and $\delta_{i}=1$ for a freely driving vehicle.

Table 2 and Figure 6 show the estimations and the observed speed distribution of only freely driving vehicles. The speed distribution of freely driving vehicles is clearly an underestimate compared with the distribution obtained by the proposed method because a vehicle with a higher desired speed tends to catch up with the leading vehicle with higher probability than one with a lower desired speed. Moreover, the estimated results of Eq. (27) vary considerably depending on the traffic volume, whereas those of the proposed method are almost the same regardless of differences in traffic volume. Because the attributes of vehicles composing the traffic flow are expected to be almost the same in these two data sets, these estimated results demonstrate the validity of the proposed estimation method. The reason why the estimation results using Eq.(27) varied is that the method does not consider that a vehicle with a higher desired speed will catch up with the leading vehicle with higher probability.

Using the estimated desired speed distribution, the platoon size distributions are estimate. Figure 7 compares the estimated and observed platoon size distributions. The estimated platoon size distribution clearly matches the observed one closely. To check the goodness of fit of the estimated platoon size distribution, a chi-square test of fitness was applied at the 5\% significance level: when $q=657$ [veh], $\chi=7.08\left(\chi_{\text {o.s }}(9)=16.92\right)$, and when $q=1,123$ [veh], $\chi=20.41\left(\chi_{\text {ous }}(13)=22.36\right)$. Thus, the estimated distribution passed the chi-square test, demonstrating that the 
platoon size distribution estimated using the observed arrival data can precisely represent the true traffic flow. It appears that this proposed method can provide a good estimate of the desired speed distribution.

\section{Application of Breakdown Probability}

The proposed method is applied to calculate the relationship between breakdown probability and traffic flow rate for a single-lane expressway where a bottleneck is located at the end of the section.

Table 2. Estimation of Desired Speed Distribution.

\begin{tabular}{|c|c|c|c|c|c|}
\hline & \multirow{2}{*}{$\begin{array}{c}\text { Traffic Volume } \\
\text { [veh] }\end{array}$} & \multicolumn{2}{|c|}{$\begin{array}{c}\text { Parameters of } \\
\text { Gumbel Distribution }\end{array}$} & \multicolumn{2}{|c|}{ Desired Speed Distribution } \\
\hline & & $\eta$ & $\mu$ & Mean $[\mathrm{km} / \mathrm{h}]$ & S.D. $[\mathrm{km} / \mathrm{h}]$ \\
\hline Speed Distribution of Freely Driving & 657 & - & - & 86.1 & 9.86 \\
\hline Vehicles & 1,123 & - & - & 84.2 & 7.93 \\
\hline Desired Speed Distribution Estimated & 657 & 89.3 & 0.085 & 96.1 & 15.1 \\
\hline by Eq. (27) & 1,123 & 94.6 & 0.069 & 103.0 & 18.6 \\
\hline Desired Speed Distribution Estimated & 657 & 89.1 & 0.095 & 95.2 & 13.5 \\
\hline by the Proposed Method & 1,123 & 90.7 & 0.097 & 96.6 & 13.2 \\
\hline
\end{tabular}

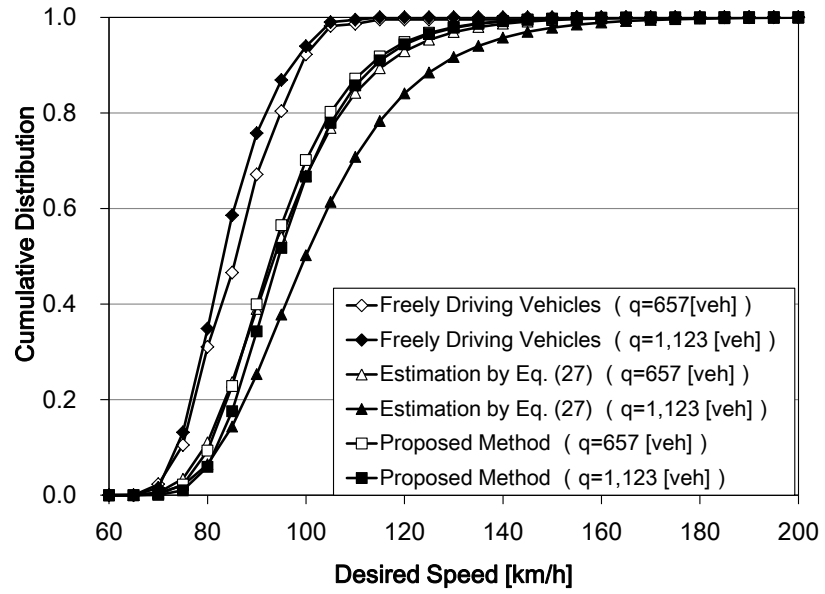

Figure 6. Estimation of Desired Speed Distribution.

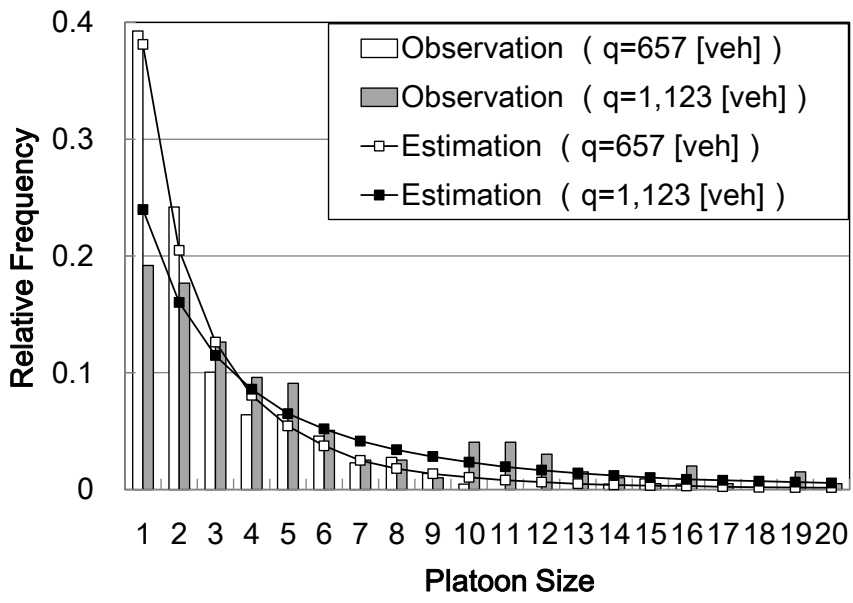

Figure 7. Estimation of Platoon Size Distribution. 


\subsection{Solution Algorithm}

To estimate the breakdown probability for a traffic flow rate by considering the randomness of vehicle arrival timing, the following approximation method is adopted.

Step 1: The traffic demand $Q$, target single-lane section length $L$, parameter vector of desired speed distribution $\boldsymbol{\beta}$, and speed transition matrix $\mathbf{P}$ are given.

Step 2: By referring to Shiomi et al. (2010), the Erlang distribution is assumed for the time headway at the entrance point of the target section. In particular, the $i$ th vehicle's entrance time $t_{i n}^{i}$ is simulated by

$$
t_{i n}^{i}=\sum_{j=1}^{i} \sum_{k=1}^{K}-\frac{1}{\lambda} \ln \left(1-R N D_{k}\right),
$$

where $R N D_{k}(k=1, \ldots, K)$ represents a uniform random number [0,1], $K$ is the phase parameter of the Erlang distribution (here, $K$ is assumed to be 2), and $\lambda$ is also a parameter of the Erlang distribution $(\lambda=Q K / 3600)$.

Step 3: Desired speeds are randomly assigned to all the vehicles based on a desired speed distribution. By the same method as that used to estimate the desired speed distribution, all platoons that formed at the end of the target section are classified according to the speed levels of the lead vehicle and the platoon size.

Step 4: By repeating Steps 2 and 3 for a sufficient simulation time T, the breakdown probability for the traffic flow rate, $p_{b d}(Q)$, is calculated as the expectation of platoon breakdown probability weighted by the length of time that each platoon occupies the bottleneck.

$$
p_{b d}(Q) \approx \sum_{i=1}^{N_{a l l}} \frac{t_{p l t}\left(v_{i}, k_{i}\right)}{\sum_{j=1}^{N_{a l l}} t_{p l t}\left(v_{j}, k_{j}\right)} \cdot p_{b d}^{p l t}\left(v_{i}, k_{i}\right),
$$

where $N_{\text {all }}$ indicates the number of platoons generated in the calculation process, and $t_{p l t}(v, k)$ is the time for which a platoon composed of $k$ vehicles and whose lead vehicle's speed is $v$ occupies the bottleneck.

\subsection{Comparison between Estimation and Observation}

To validate the estimated breakdown probability for a traffic flow rate, the estimated relationship between breakdown probability and traffic flow rate is compared with the observed breakdown flow rate, which is defined as the traffic volume during $15[\mathrm{~min}]$ just before the 5 -min average speed becomes less than $50[\mathrm{~km} / \mathrm{h}]$.

The observations were made at $48.4 \mathrm{kp}$ of the inbound lane of the Tokai-Hokuriku expressway, as shown in Figure 8, which is single-lane section, from March 1, 2003 to November 30, 2003. The data were collected by loop detectors, which were installed just behind the entrance to Kariyasu Tunnel, where congestion queues often appear. The single-lane section just behind the bottleneck is $2.3-\mathrm{km}$ long. The relationship between the 5 -min flow rate and 5 -min average speed at $48.4 \mathrm{kp}$ is shown in Figure 9.

To estimate the breakdown probability, the desired speed distribution is assumed to be a Gumbel distribution, and its parameters are set to $(\eta, \mu)=(90.7,0.097)$ following the estimation results in section 4 . For simplicity, the time headway of a vehicle in a platoon is assumed to be the inverse number of the observed maximum 15-min traffic flow rate at $48.4 \mathrm{kp}$. Because the maximum flow rate was $336 \mathrm{veh} / 15 \mathrm{~min}$, the time headway was set to 2.68 [s] regardless of the speed. Thus, the time duration $t_{p l t}(v, k)$ is derived as

$$
t_{p l t}(v, k)=2.68 \cdot(k-1) \text {. }
$$

It implies that the traffic capacity is set to 1,344 [veh/h]. If the traffic demand exceeds $1,344[\mathrm{veh} / \mathrm{h}]$, the traffic breakdown probability is expected to be 1.0. A speed transition matrix is generated on the basis of the observations of the single-lane section of the Tokai-Hokuriku expressway described in 3.3.1, as follows, where the criteria for the speed levels are set to $V_{0}=50[\mathrm{~km} / \mathrm{h}], V_{1}=60[\mathrm{~km} / \mathrm{h}], \ldots, V_{6}=110[\mathrm{~km} / \mathrm{h}]$. That is, in this case study, when the speeds of vehicles in a platoon decrease to less than $50[\mathrm{~km} / \mathrm{h}]$, traffic breakdown is defined to have occurred. The simulation time $T$ is set to 1,000 hours. 


$$
\mathbf{P}=\left(\begin{array}{cccccccc}
1 & 0 & 0 & 0 & 0 & 0 & 0 & 0 \\
0.1094 & 0.7813 & 0.0938 & 0.0156 & 0 & 0 & 0 & 0 \\
0.0054 & 0.0435 & 0.6467 & 0.2826 & 0.0163 & 0.0054 & 0 & 0 \\
0 & 0.0010 & 0.0714 & 0.6945 & 0.2231 & 0.0080 & 0.0020 & 0 \\
0 & 0.0006 & 0.0006 & 0.1585 & 0.6972 & 0.1300 & 0.0093 & 0.0037 \\
0 & 0 & 0 & 0.0026 & 0.2718 & 0.5910 & 0.1240 & 0.0105 \\
0 & 0 & 0 & 0 & 0.0465 & 0.2957 & 0.5615 & 0.0963 \\
0 & 0 & 0 & 0 & 0.0080 & 0.0560 & 0.3520 & 0.5840
\end{array}\right)
$$

For comparison, the stochastic capacity is also estimated by the product limit method (PLM) proposed by Brilon et al. (2005). In the PLM, the parameters of the distribution functions are estimated by applying a maximum likelihood technique. The likelihood function is given by

$$
L(\boldsymbol{\omega})=\prod_{i=1}^{n} f_{c}\left(q_{i} \mid \boldsymbol{\omega}\right)^{\delta_{i}} \cdot\left[1-F_{c}\left(q_{i} \mid \boldsymbol{\omega}\right)\right]^{1-\delta_{i}},
$$

where

$\omega=$ parameter vectors specifying the distribution functions,

$f_{c}\left(q_{i} \mid \boldsymbol{\omega}\right)=$ stochastic density function of capacity. In this study, the Weibull distribution is applied.

$F_{c}\left(q_{i} \mid \boldsymbol{\omega}\right)=$ cumulative distribution function of capacity

$n$ = number of intervals

$\delta_{i}=\left\{\begin{array}{l}1, \text { if uncensored }\left(q_{i} \text { induced the traffic breakdown }\right) \\ 0, \text { otherwise }\end{array}\right.$

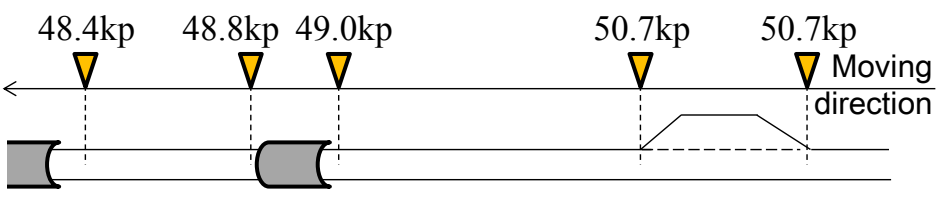

Kariyasu TN

Figure 8. Data Collection Site at $48.4 \mathrm{kp}$ of the Tokai-Hokuriku Expressway.

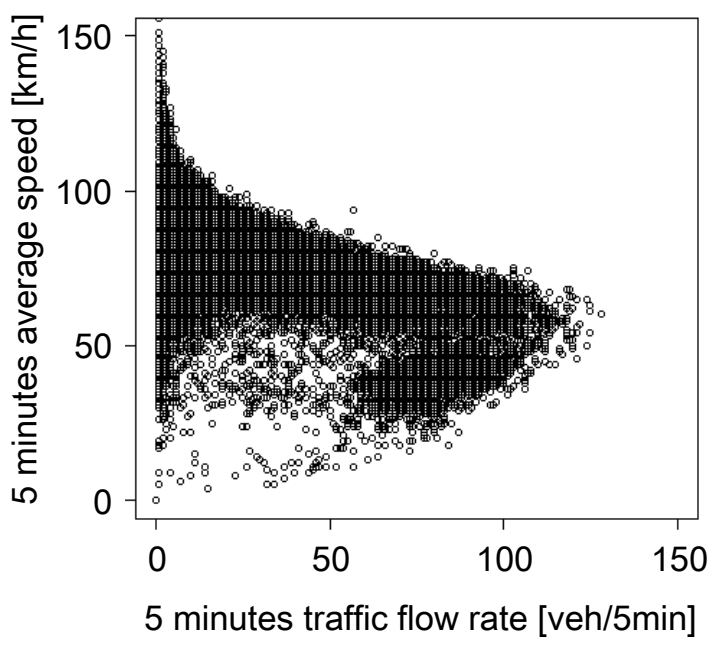

Figure 9. Fundamental Relationship between 5-Min Traffic Flow Rate and 5-Min Average Speed. 


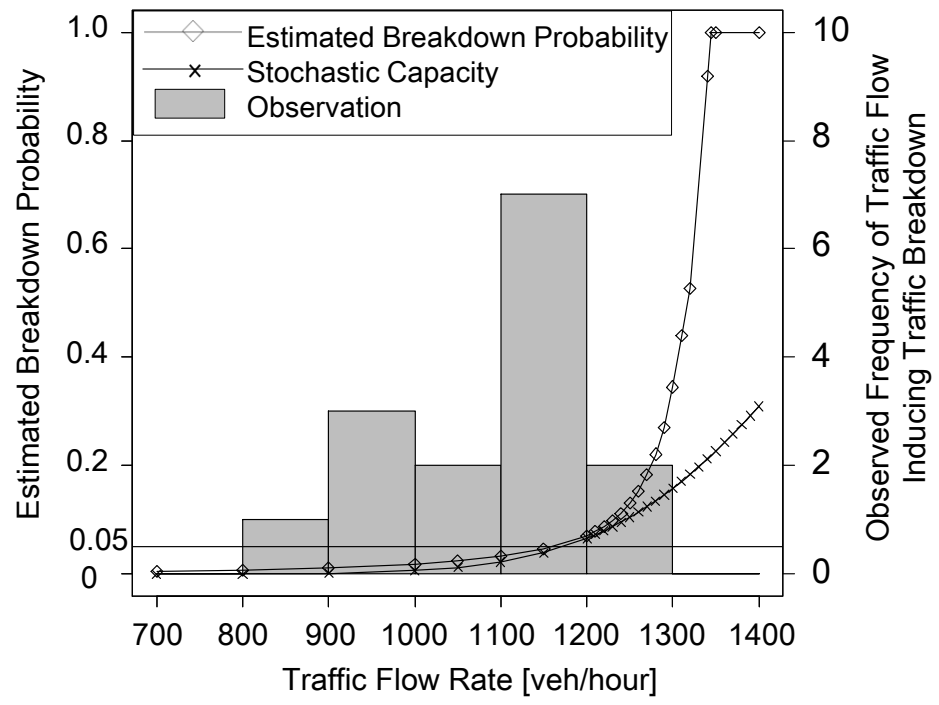

Figure 10. Comparison of Estimated Breakdown Probability, Estimated Stochastic Capacity and Observed Breakdown Flow Rate. The Weibull distribution is specified by two parameters; the shape parameter, $\kappa$, and the scale parameter, $\lambda$. The estimations of the stochastic capacity are $\omega=(\kappa, \lambda)=(14.16,1431.15)$.

The estimation results and observed breakdown flow rate are illustrated in Figure 10. The breakdown flow rate is widely distributed between $800[\mathrm{veh} / \mathrm{h}]$ and $1,300[\mathrm{veh} / \mathrm{h}]$, and the breakdown probability estimated by the proposed method increases monotonously; when the traffic flow rate is larger than around 1,350 [veh/h], it reaches 1.0 . In contrast, the stochastic capacity is about 0.3 at a traffic flow rate 1,400 [veh/h], although such a high flow rate never appears. This result indicates that the stochastic capacity tends to overestimate the traditionally defined traffic capacity. It is notable that at the mode of the observed frequency distribution $[1,100,1,200)$, both the breakdown probability and the stochastic capacity are around 0.05 . The reason is considered to be that the breakdown probability increases as the traffic flow rate increases, but at the same time, the probability that the traffic flow rate is observed decreases. That is why the observed frequency takes the highest value at a breakdown probability and stochastic capacity of 0.05 . On the basis of these discussions, it can be concluded that the estimated breakdown probability is valid with respect to the observed frequency of the breakdown flow rate and stochastic capacity estimated by Brilon et al. (2005).

\subsection{Sensitivity of Breakdown Probability to Traffic Flow Rate}

In this section, the factors affecting the breakdown probability are investigated.

Figure 11 depicts the relationship between traffic flow rate and breakdown probability with respect to single-lane section length. In this analysis, traffic flow rate varies from 800 [veh/h] to $1,400[\mathrm{veh} / \mathrm{h}]$, and the single-lane section length is set to $2.5[\mathrm{~km}], 5.0[\mathrm{~km}]$ or $10.0[\mathrm{~km}]$. As the traffic flow rate increases, the traffic breakdown probability clearly increases as well; when the demand exceeds around 1,350 [veh/h], the breakdown probability is close to 1.0 . As the single-lane section length increases, the breakdown probability curve moves upward on the graph. This implies that the longer the single-lane section is, the more frequently traffic breakdown occurs. In other words, at the same values of the traditional traffic capacity, the practical traffic capacity (i.e., the observed traffic volume just before traffic breakdown) tends to decrease as the single-lane section length increases. Yoshikawa et al. (2008) reported this tendency, which confirms the validity of the proposed estimation method.

To investigate the influence of the desired speed distribution on the breakdown probability, Figures 12 and 13 illustrate the breakdown probability curves for various desired speed distributions. The mean and standard deviation of a Gumbel distribution with $(\eta, \mu)=(90.7,0.097)$ correspond to $96.6[\mathrm{~km} / \mathrm{h}]$ and $13.2[\mathrm{~km} / \mathrm{h}]$, respectively. Apart from this distribution, we also examined two other speed distributions obtained by keeping the standard deviation constant at $13.2[\mathrm{~km} / \mathrm{h}]$ and set the mean as $90.0[\mathrm{~km} / \mathrm{h}]$ and $110.0[\mathrm{~km} / \mathrm{h}]$, where the parameters of the Gumbel distribution were $(\eta, \mu)=(74.1,0.097)$ and $(\eta, \mu)=(104.1,0.097)$, respectively. Figure 12 shows the breakdown probability curves for these three distributions. In addition, we examined the effect of changing the standard 
deviation by keeping the mean constant at $96.6[\mathrm{~km} / \mathrm{h}]$ and set the standard deviation as $5.0[\mathrm{~km} / \mathrm{h}]$ and $15.0[\mathrm{~km} / \mathrm{h}]$, where the parameters of the Gumbel distribution were $(\eta, \mu)=(94.4,0.257)$ and $(\eta, \mu)=(87.7,0.064)$, respectively. Figure 13 shows the breakdown probability curves for these two distributions and for the Gumbel distribution with $(\eta, \mu)=(90.7,0.097)$. These figures show that as the mean of the desired speed distribution decreases and the standard deviation increases, the breakdown probability curve moves upward. That is, traffic breakdown occurs more frequently, and the practical traffic capacity decreases, because large platoons are more likely to be generated. Thus, this investigation suggests that the occurrence of traffic breakdown must be reduced by controlling the desired speed distribution of traffic flow. For example, a dynamic variable speed limit that encourages slow vehicles to speed up and fast vehicles to slow down and an adaptive cruise control system that can disperse platoons should be effective measures of breakdown prevention.

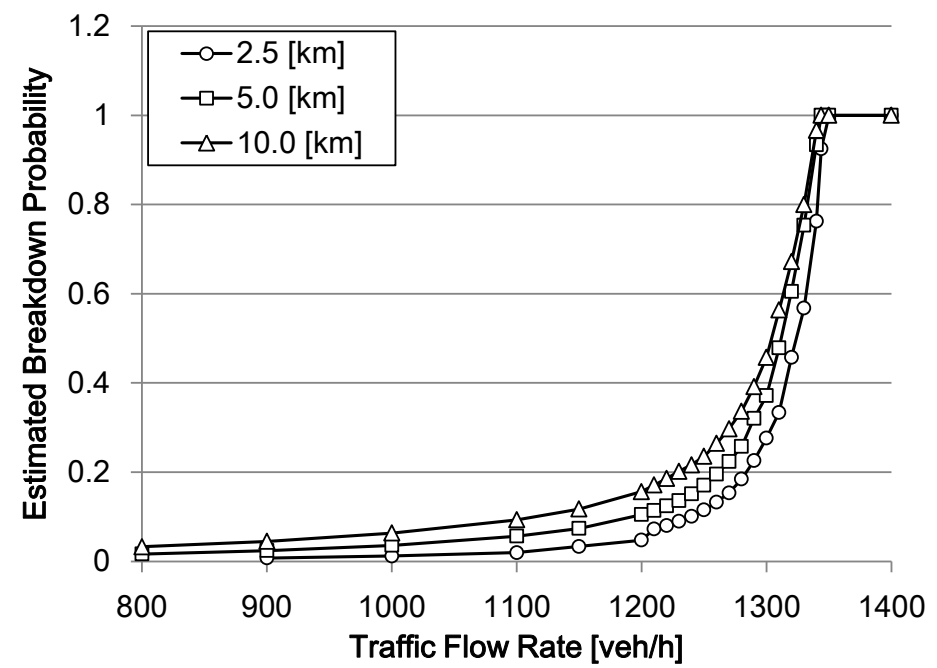

Figure 11. Variation in Breakdown Probability with Respect to Single-Lane Section Length. The target singlelane section length is either $2.5[\mathrm{~km}], 5.0[\mathrm{~km}]$, or $10.0[\mathrm{~km}]$.

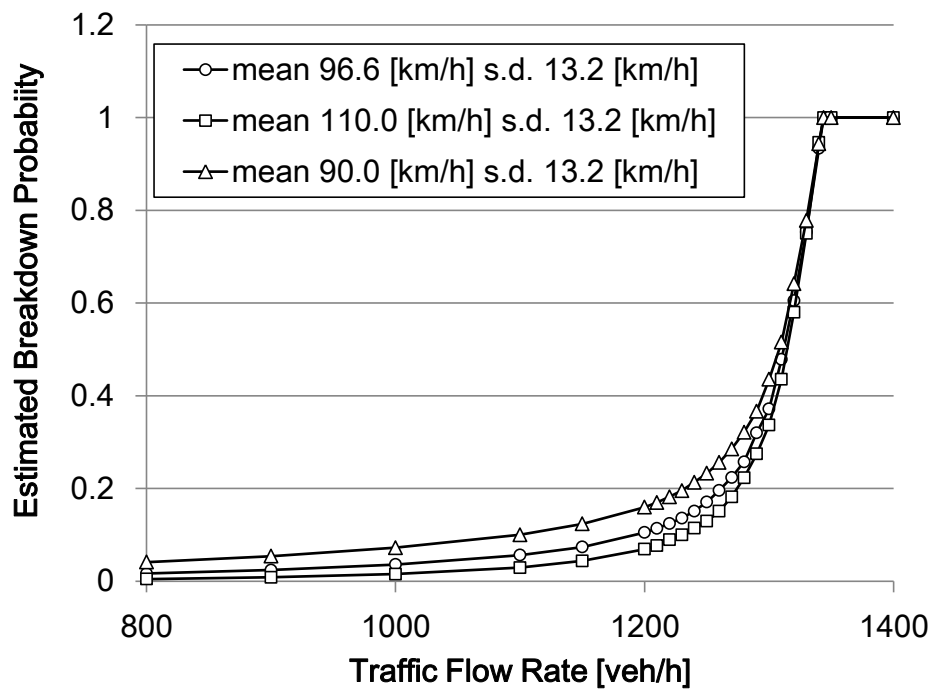

Figure 12. Variation in Breakdown Probability with Respect to Mean of Desired Speed Distribution. The standard deviation is kept constant, and the mean of the desired speed distribution is either $90.0[\mathrm{~km} / \mathrm{h}], 96.6[\mathrm{~km} / \mathrm{h}]$, or 110.0 $[\mathrm{km} / \mathrm{h}]$. 


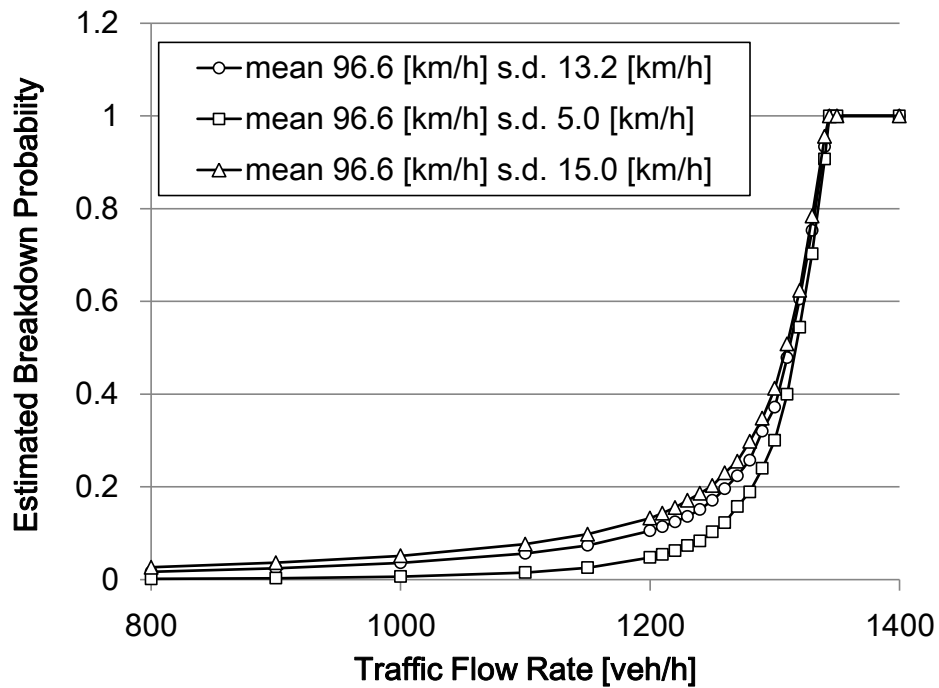

Figure 13. Variation in Breakdown Probability with Respect to S.D. of Desired Speed Distribution. The mean was kept constant, and the standard deviation of the desired speed distribution was either $5.0[\mathrm{~km} / \mathrm{h}], 13.2[\mathrm{~km} / \mathrm{h}]$, or $15.0[\mathrm{~km} / \mathrm{h}]$.

\section{Conclusion}

The present study investigated the mechanism of traffic breakdown at a bottleneck on an expressway. A platoonbased traffic flow model was developed on the assumption that the stochastic breakdown mechanism comprises two types of stochastic processes: platoon formation, which is caused by heterogeneity in driving behaviors such as desired speeds and can be regarded as a metastable traffic state in which a deceleration wave can propagate, and the speed transition process within a platoon.

After platoons were defined with respect to the speed correlation between two successive vehicles, a platoon formation model was proposed by focusing on the desired speed distribution. Next, on the basis of Koshi's conjecture about the breakdown mechanism and an investigation of relative speed in a platoon, a speed transition model was developed by applying an absorbing Markov chain model. Then, the traffic breakdown probability was rigorously defined, and calculation methods were proposed. This proposed model features the following points: it can deal with the stochastic nature of traffic flow and heterogeneity among vehicles, and can also be calibrated using only fixed-point observation data. After these proposed models were validated using the observed traffic flow data, they were applied to a simple one-way, one-lane expressway section containing a bottleneck, and the stochastic nature of traffic breakdown was demonstrated through theoretical calculations. The results revealed that the breakdown probability increases as the single-lane distance from the entrance to the bottleneck section increases, the mean of the desired speed distribution decreases, and the variance of the desired speed distribution increases. The investigation implied that the breakdown probability can be reduced by instituting measures regulating the desired speed distribution because uncertainty in traffic flow phenomena may be caused by heterogeneity in driving behavior or vehicle performance. If this is true, a traffic management measure that controls the variability in each vehicle would have a significant effect. For example, an adaptive cruise control system or, at least, a regulation relating to the maximum and minimum speed limitation would reduce the occurrence of traffic breakdown and improve the efficiency of expressways.

The proposed model considers only the transition from the free-flow state to the congested state based on fixedpoint observations. However, traffic congestion changes dynamically and spatially. In future studies, the model should be expanded to deal with the dynamics of traffic flow, including the situation after a traffic breakdown. Then, a method of estimating the uncertainty in travel time and the level of service on expressways can certainly be developed. Other future topics of the study are as follows: (i) The Gumbel distribution is assumed for the desired speed distribution, but the suitability of various distribution functions for the desired speed distribution should be considered more carefully. (ii) For simplicity, the time headway of a vehicle in a platoon was assumed to be constant regardless of the traffic state. To investigate traffic breakdown more precisely, fluctuations in time 
headway should be analyzed in detail. (iii) Although the speed transition is assumed to be described by a Markov chain model, the details of the speed transition are considered to be more complicated and requiring further investigation. (iv) The model can currently be applied only to a single-lane section. It should be expanded to depict traffic phenomena on multilane expressways, including lane-changing and overtaking maneuvers, and other types of bottlenecks such as merging sections.

\section{Acknowledgments}

The authors are grateful to Professor Masao Kuwahara at Tohoku University for his useful comments to the detail of this study, anonymous referees for their constructive comments to an earlier version of this paper, which aided in improving the quality of the paper, and both of the engineers at Central Nippon Expressway Co. Ltd. and at Express Highway Research Foundation of Japan, who provided the data set for the analysis.

Professor Ryuichi Kitamura passed away on February19, 2009. He was a member of the International Advisory Committee of ISTTT. This research is the last one which he had made with us. We missed the great master who had told us the way scholars should go. We want to express sincere thanks and to pray for the repose of his soul.

\section{References}

Anderson, T.W., Goodman, L.A., 1957. Statistical inference about Markov chains. The Annals of Mathematical Statistics 28(1), 89-110.

Bennett, C.R., Dunn, R.C.M., 1994. Critical headway on two-lane highways in New Zealand. Proceedings of Second Symposium on Highway Capacity, 91-100.

Botma, H., Bovy, P.H.L., 2001. Free speed distribution at arterial highways: estimation approach. Proceedings of 9th WCTR, CD-ROM.

Branston, D., 1979. A method of estimating the free speed distribution for a road. Transportation Science 13(2), 130-145.

Brilon, W., Geistefeldt, J., Regler, M., 2005. Reliability of freeway traffic flow: a stochastic concept of capacity. In: Mahmassani,H.S. (Ed.), Proceeding of the 16th International Symposium on Transportation and Traffic Theory. Elsevier, Maryland, 125-143.

Daganzo, C.F. Cassidy, M.J., Bertini, R.L., 1999. Possible explanation of phase transitions in highway traffic. Transportation Research Part A 33(5), 365-379.

Daganzo, C.F., Laval, J.A., 2005. On the numerical treatment of moving bottleneck. Transportation Research Part B 39(1), 31-46.

Elefteriadou, L., Roess, L., McShane, W.R., 1995. Probabilistic nature of breakdown at freeway merge junctions. Transportation Research Record 1484, 80-89.

Gazis, D.C., Herman, R., 1992. The moving and "phantom" bottleneck. Transportation Science 26, 223-229.

Geistefeldt, J., Brilon, W., 2009. A comparative assessment of stochastic capacity estimation methods. In Lam, W.H.K., Wong, S.C., Lo, H.K. (Eds.), Proceeding of the 18th International Symposium on Transportation and Traffic Theory, Springer, 125-143.

Gibbs, P.G., 1977. A queuing model for traffic flow. Journal of the Royal Statistical Society Series B 39(2), $276-282$.

HCM, 2000. Highway Capacity Manual 2000. Transportation Research Board. National Academy of Sciences, Washington, DC.

Hoogendoorn, S.P., 2005. Unified approach to estimating free speed distribution. Transportation Research Part B 39(8), 709-727.

Kerner, B.S., Rehborn, H., 1997. Experimental property of phase transition in traffic flow. Physics Review Letter 79, 4030.

Koshi, M., 1986. Capacity of motorway bottlenecks. Journals of the Japan Society of Civil Engineers 371(IV-5), 1-7 (in Japanese).

Koshi, M., Iwasaki, M., Ohkura, I., 1983. Some findings and an overview on vehicular flow characteristics. In: Hurdle, V. F., Hauer, E., Steuart, G. N. (Eds.), Proceedings of the eighth International Symposium on Transportation and Traffic Theory, University of Toronto Press, Toronto, 403-451.

Kühne, R. Anstett, N., 1999. Stochastic method for analysis of traffic pattern formation. Proceedings of the 14th International Symposium on Transportation and Traffic theory. Jerusalem. 
Kühne, R., Mahnke, R., 2005. Controlling traffic breakdowns. In: Mahmassani,H.S. (Ed.), Proceedings of 16th Transportation and Traffic Theory. Elsevier, Maryland, 229-244.

Kühne, R., Mahnke, R., Hinkel, J., 2007. Understanding traffic breakdown: A stochastic approach. In: Allsop, R.E., Bell, M.G.H., Heydecker, B.G. (Eds.), Proceedings of the 17th Transportation and Traffic Theory. Elsevier, Amsterdam, 777-789.

Lay, M.G., 1986. Handbook of road technology. Gordon and Breach.

Laval, J.A., 2006. Stochastic processes of moving bottlenecks: Approximate formulas for highway capacity. Transportation Research Record 1988, 86-91.

Lorenz, M., Elefteriadou, L., 2000. A probabilistic approach to defining freeway capacity and breakdown. Proceeding of the fourth International Symposium on Highway Capacity, 84-95.

Mahnke, R., Kaupužsb, J., Lubashevskyc, I., 2005. Probabilistic description of traffic flow. Physics Reports 408 (12), $1-130$.

Miller, A.J., 1961. A queuing model for road traffic flow. Journal of the Royal Statistical Society B23, 64-75.

Minderhoud, M.M., Botma, H., Bovy, P.H.L., 1997. Roadway capacity estimation methods explained and assessed. Transportation Research Record 1572, 59-67.

Newell, G.F., 1998. A moving bottleneck. Transportation Research part B 32(8), 531-537.

Oguchi, T., Katakura, M., Shikata, S., 2001. An empirical study on characteristics of traffic breakdown at bottlenecks on a basic motorway section. Expressways and Automobiles 44 (12), 27-34. (in Japanese)

Okamura, H., Watanabe, S., Watanabe, T., 2000. An empirical study on the capacity of bottlenecks on the basic suburban expressway sections in Japan. Proceeding of the fourth International Symposium on Highway Capacity, 120-129.

Shiomi, Y., Yoshii, T., Kitamura, R., 2010. A method of estimating platoon size distribution based on desired speed distribution. Traffic Engineering 45 (1), 58-67. (in Japanese).

Surasak, T., Okura, I., Nakamura, F. 2001. Study of platoon characteristics on a multi-lane expressway. Journal of Infrastructure Planning Review 18(5), 909-917.

Tamura, Y., Chisyaki, T. 1987. Modeling and study of speed and bunch distributions considering fluctuations of traffic flow. Proceedings of the 10th International Symposium on Transportation and Traffic Theory. Cambridge. U.S.A., pp. 99-118.

Tanner, J.C., 1961. A derivation of the Borel distribution. Biometrika 48, 222-224.

Yoshikawa, R., Shiomi, Y., Yoshii, T., Kitamura, R., 2008. A study on bottleneck capacity of two-lane expressways. Traffic Engineering 43(5), 48-58. (in Japanese) 